\title{
MMP-9 regulates CX3CL1/CX3CR1 in the early phase of neuropathic pain in chronic sciatic nerve constriction injury $(\mathrm{CCl})$ rats
}

\author{
Li Zhao^, Congzhong Song, Yaqin Huang, Weiping Lei, Jianliang Sun \\ Department of Anesthesiology, Affiliated Hangzhou First People's Hospital, Zhejiang University School of Medicine, Hangzhou, China \\ Contributions: (I) Conception and design: L Zhao; (II) Administrative support: J Sun; (III) Provision of study materials or patients: Y Huang; (IV) \\ Collection and assembly of data: C Song; (V) Data analysis and interpretation: L Zhao; (VI) Manuscript writing: All authors; (VII) Final approval of \\ manuscript: All authors. \\ Correspondence to: Jianliang Sun. Department of Anesthesiology, Affiliated Hangzhou First People's Hospital, Zhejiang University School of Medicine, \\ Hangzhou, China. Email: sj16805@zju.edu.cn.
}

Background: To observe the effects of MMP-9 (matrix metalloproteases-9) on the mechanical allodynia and thermal hyperalgesia and the expression of CX3CL1 (CX3C chemokine ligand 1) protein in the spinal dorsal root ganglion (DRG) in rats with chronic sciatic nerve constriction injury (CCI).

Methods: A total of 84 male SD rats were randomly divided into seven groups, namely the normal group , the sham operation group, the model group, the CCI + MMP-9 group, the CCI + TIMP-1 group , the CCI + siRNA group, and the CCI + MM-siRNA group. The CCI model was prepared 5 days after implantation of intrathecal catheter. The rat paw mechanical withdrawal threshold (PWMT) and paw thermal withdrawal latency (PWTL) were measured 1 day before CCI surgery and 1, 2, 3 and 5 days after CCI respectively. Western blot (WB) was used to detect the expressions of the MMP-9 and the CX3CL1 protein in the L5 DRG of the spinal cord 1 day after CCI operation.

Results: (I) Behavioral assessment of hyperalgesia: compared with the Sham group, the PWMT and PWTL of the CCI group were significantly reduced at each time point after CCI surgery; compared with the CCI group, the PWMT and PWTL of the CCI + MMP-9 group decreased 1 day after CCI; for the PWMT and PWTL of the CCI + TIMP-1 group and CCI + siRNA group, PWMT and PWTL increased 1 day after CCI; (II) the expressions of MMP-9 and CX3CL1 protein in the DRG of the spinal cord: compared with Sham group, the expressions of MMP-9 and CX3CL1 protein in the DRG of the CCI group increased significantly 1 day after CCI surgery; compared with the CCI group, the expressions increased in the CCI + MMP-9 group 1 day after CCI . However, the expressions of MMP-9 and CX3CL1 in the CCI + TIMP-1 group and CCI + siRNA group were reduced on the first postoperative day.

Conclusions: The mechanism of MMP-9 participating in the early phase of neuropathic pain (NP) in CCI rats is related to the upregulation of CX3CL1.

Keywords: MMP-9; CX3CL1; chronic sciatic nerve constriction injury (CCI); neuropathic pain (NP)

Submitted Apr 13, 2020. Accepted for publication Jul 04, 2020.

doi: 10.21037/apm-20-1078

View this article at: http://dx.doi.org/10.21037/apm-20-1078

^ ORCID: 0000-0001-6597-8067. 


\section{Introduction}

Neuropathic pain (NP) is a kind of intractable pain arising as a direct consequence of a lesion or disease affecting the somatosensory system (1). Because its pathogenesis is not fully understood, the current treatment of NP is mostly limited to temporary pain relief through peripheral or central block of nerve transmission, and there is no specific target treatment or method. Therefore, in-depth research on the pathogenesis of NP, especially the molecular mechanism, will help find effective therapeutic targets and drugs, so as to increase the efficacy of NP treatment and the quality of life of NP patients. It is known that MMP-9 is a kind of proteolytic enzyme, which can not only hydrolyze extracellular matrix, but can also act on a variety of chemokines, such as SDF-1 (stromal cell derived factor-1), SCF (stem cell factor), and play an important role in inflammatory response, angiogenesis and other pathophysiological processes (2). It has been found that MMP-9 may be critically involved in the early stage of NP, but the way in which MMP-9 plays its role is still unclear (3). In our previous study, we found that under the condition of NP, CX3CL1/CX3CR1 (CX3C chemokine receptor 1) can activate the spinal microglia ERK5 (extracellular signal-regulated kinase 5), which is involved in the process of spinal pain signal transduction $(4,5)$, but the upstream mechanism of CX3CL1 is still unknown. The purpose of this study was to observe the changes of pain behavior and the expressions of MMP-9 and CX3CL1 protein in the dorsal root ganglion (DRG) of the spinal cord after intrathecal injection of MMP-9 agonists and inhibitors, small interfering RNA (siRNA) gene-silencing, and interference technology knock-out of MMP-9 expression in chronic sciatic nerve constriction injury (CCI) rats. We also planned to explore whether MMP-9 participates in the early stage of NP in CCI rats by regulating the expression of CX3CL1 protein, so as to provide a theoretical basis for finding new therapeutic targets and intervention drugs for NP in clinic.

We present the following article in accordance with the ARRIVE reporting checklist (available at http://dx.doi. org/10.21037/apm-20-1078).

\section{Methods}

\section{Animals and reagents}

Eighty-four specific pathogen-free (SPF) male SpragueDawley (SD) rats weighing 190-210 g were purchased from the Animal Experimental Center, Zhejiang Chinese Medical University, China. The procedures of animal experiments were performed by Guide for the Care and Use of Laboratory Animals and were approved by Zhejiang Chinese Medical University. All surgical procedures were performed under general anesthesia, and every effort was made to minimize stress. Animals were housed in standard cage in a temperature-controlled $\left(22 \pm 2{ }^{\circ} \mathrm{C}\right)$ colony room under a $12 \mathrm{~h} \mathrm{light/dark} \mathrm{cycle} \mathrm{regime,} \mathrm{with} \mathrm{food} \mathrm{and} \mathrm{water}$ available.

The MMP-9 and MMP-9 rabbit polyclonal antibodies were purchased from Chemicon; tissue inhibitors of matrix metalloendopeptidases-1 (TIMP-1) were purchased from Millipore; MMP-9 siRNA sequence (sense: 5'-GACUUGCCGCGAGACAUGAtt-3', antisense: 3 '-ttCUGAACGGCGCUCUGUACU-5') and MMP-9-MM siRNA sequence (sense, 5'-GACUUCGCGCGAGACAUGAtt-3'; antisense, 3'-ttCUGAAGCGCGCUCUGUACU-5') were provided by Hangzhou Ribobio. CX3CL1 rabbit polyclonal antibody was purchased from Genetex.SDS-PAGE and diaminobenzidine (DAB) developer were purchased from Beijing Zhongshan Golden Bridge Company, China.

\section{Experimental process}

\section{Implantation of intrathecal catheter}

According to the improved method of Yang (6), a PE10 catheter (depth $2 \mathrm{~cm}$ ) was inserted caudally from the cistern magna and advanced $7.0-7.5 \mathrm{~cm}$ to the level of the lumbar enlargement. Proper location was confirmed by a temporary motor block of both hind limbs after injection of $20 \mu \mathrm{L}$ $2 \%$ lidocaine. Only animals with no evidence of neurologic deficit after the operation were studied. After the catheter was successfully placed, the rats were raised in a single cage, and the CCI surgery was performed 5 days later (7).

\section{CCI model surgery}

Rats were injected intraperitoneally with $10 \%$ chloral hydrate $(300 \mathrm{mg} / \mathrm{kg})$. After the rats entered the deep anesthesia state, the left common sciatic nerve of the rat was isolated and exposed. Four ligatures (4.0 chromic catgut) threaded at four sites with approximately $1 \mathrm{~mm}$ intervals were loosely tied around the nerve proximally to the sciatic trifurcation in a fashion that did not block the supply of nerve blood flow (8). In the Sham group, only the nerves were exposed and not ligated. When rats showed typical signs of NP such as weight-bearing reduction of the hind 
limb, contracture of the paw, licking, and no motor function limitation such as lameness, they were judged as successful models.

\section{Experimental grouping}

A total of $84 \mathrm{SD}$ rats were divided into seven groups using a random number table, with 12 rats in each group. The groups are described below.

Con group: no surgery and no injection; Sham group: only exposure of the sciatic nerve and no ligation; CCI group: prepared for surgery with no injection; CCI + MMP-9 group: intrathecal injection of MMP-9 agonist 1 pmol/10 $\mu \mathrm{L}$, immediately after CCI; CCI + TIMP-1 group: intrathecal injection of MMP-9 inhibitor $4 \mathrm{pmol} /$ $10 \mu \mathrm{L}$, immediately after CCI; CCI + siRNA group and CCI + MM-siRNA group: intrathecal injection of MMP-9 gene-silencing and MMP-9 mismatch gene-silencing control group $2 \times 5 \mu \mathrm{g} / 10 \mu \mathrm{L}$, started 2 days before CCI.

\section{Behavioral tests}

The PWMT and PWTL of the rat's hind foot were measured at 1 day before and 1, 2, 3 and 5 days after CCI.

The procedure for PWMT measurement was as follows. First, the rat was placed on a wire fence of $11.5 \mathrm{~cm} \times 17 \mathrm{~cm} \times$ $14 \mathrm{~cm}$ to adapt to the environment. After the rat was quiet for about 15 minutes, the filament was aligned with a diameter of about $0.5 \mathrm{~mm}$ on the instrument to the plantar side of the rat (between the 4 foot pads), and stimulated at $2.5 \mathrm{~g} / \mathrm{s}$, with the maximum stimulation amount of $50 \mathrm{~g}$. If the rat's foot was rapidly contracted after stimulation, the strength of the rat's foot contraction (unit: $g$ ) was considered to be the mechanical pain threshold. Each rat was measured 3 times with an interval of 3 to 5 minutes, and the average was taken as the final PWMT.

The procedure for PWTL measurement was as follow. The rats were placed in a transparent plexiglass box, and after a wait of about 15 minutes for the rats to stop selfgrooming and exploratory activity, the e a mark was placed on the tester in the center of the plantar side of the rat, with the foot pad being avoided. The time from the start to the appearance of leg-raising in rats was used as the rat's thermal pain threshold (unit: seconds). Each foot was irradiated 3 times at 5 -minute intervals, with the average being taken as the rat's final PWTL. In order to prevent the rats from being scalded by heat radiation, we set the upper limit of the measurement time to 20 seconds (9).

\section{Western blot analysis}

One day after CCI, the pain threshold of the rats was considered met, and $10 \%$ chloral hydrate $(300 \mathrm{mg} / \mathrm{kg})$ was injected intraperitoneally. The head was fixed on the operation table, the rat's abdomen was quickly cut, and the xiphoid was clamped with hemostats. Scissors were used to cut along the left and right sides of the sternum to fully expose the thoracic cavity. Intravenously, $200 \mathrm{~mL}$ of $0.9 \%$ physiological saline was perfused at a constant rate. After the right atrial appendage exudate became pale red and rat muscle tremor appeared, the hind limbs became white and the tail became straight, indicating successful perfusion. The DRG of the spinal lumbar segment (all operations were performed on the ice box) was extracted, and quickly put it into a liquid nitrogen bottle for storage. The proteins of DRG tissues from the spinal and lumbar spinal segments of each group of rats were extracted to make protein samples, and the proteins were separated by SDSPAGE electrophoresis. The nitrocellulose membrane was transferred to a membrane, and the current was transferred at $30 \mathrm{~mA}$ at $4{ }^{\circ} \mathrm{C}$ overnight. After blocking was completed, primary and secondary antibodies were added and incubated at room temperature for 1.5 and 1 hour, respectively. Detection was conducted by chemiluminescence method. After binding with luminescent substrate, the protein samples was developed by $\mathrm{X}$-ray exposure, stored, and analyzed by ImageJ.

\section{Statistical analysis}

All data were statistically processed using SPSS 17.0 software. Measurement data for normal distribution are expressed as mean \pm standard deviation (mean $\pm \mathrm{SD}$ ). The $t$-test was used to compare the data between the two groups, the one-way analysis of variance (ANOVA), was used to compare the multiple groups, and the SNK-q test was used to compare the two pairs. A $\mathrm{P}$ value $<0.05$ was considered statistically significant.

\section{Results}

\section{Changes in pain threshold of rats in each group}

The postoperative condition of CCI rats was generally good, no autophagic limb phenomenon occurred and typical spontaneous hyperalgesia gradually appeared. The rats left limbs were affected, and were dragged or suspended, and there was clear walking and lameness. 

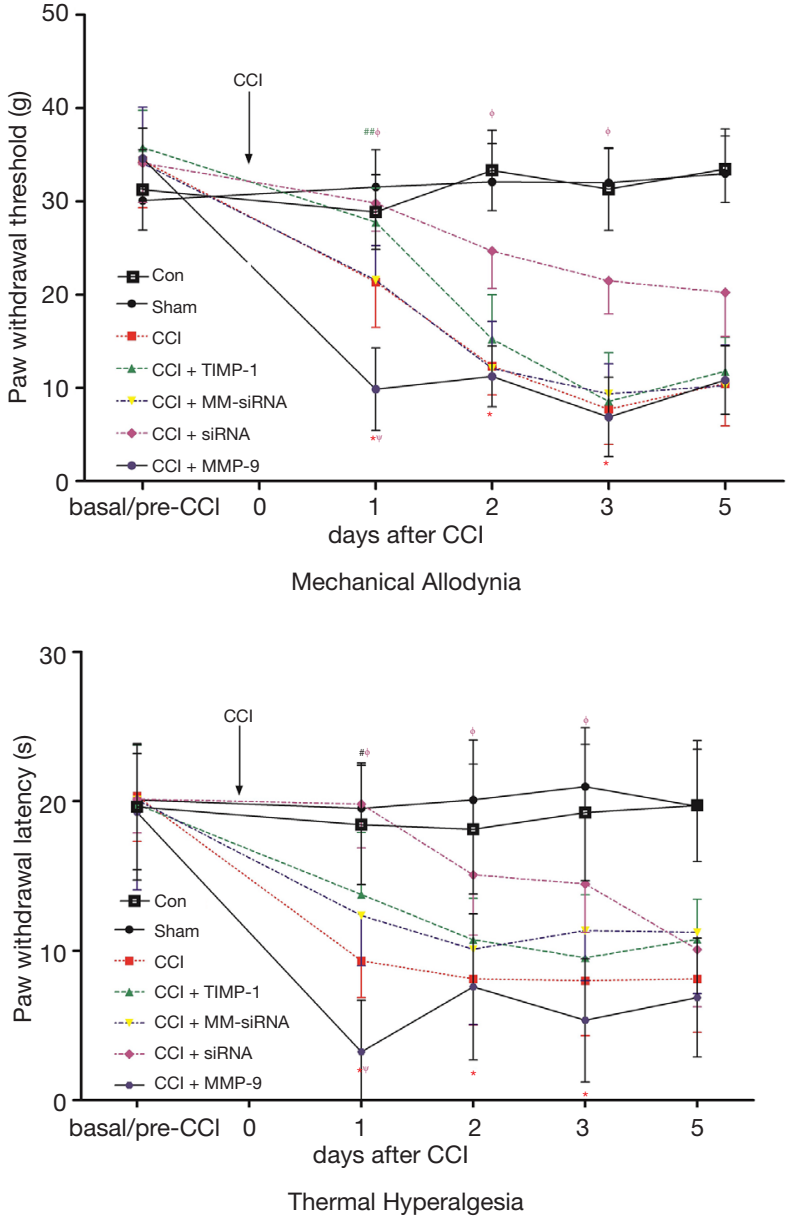

Figure 1 Trend graph of pain threshold of rats in each group over time. The data are presented as mean \pm SD in each group $(n=6)$. *, $\mathrm{P}<0.01 \mathrm{CCI}$ vs. Sham group; ${ }^{*}, \mathrm{P}<0.01 \mathrm{MMP}-9$ vs. CCI group; \#\#, $\mathrm{P}<0.01$ and ", $\mathrm{P}<0.05$ TIMP-1 vs. CCI group; ${ }^{\phi}, \mathrm{P}<0.01 \mathrm{CCI}$ + siRNA vs. CCI group. CCI, chronic sciatic nerve constriction injury.

There was no statistical difference in the basal values of PWMT and PWTL in each experimental group $(\mathrm{P}>0.05)$. Compared with the Sham group, the PWMT and PWTL of the CCI group were significantly reduced at each time point after surgery $(\mathrm{F}=32.71, \mathrm{P}=0.000 ; \mathrm{F}=14.65, \mathrm{P}=0.000)$; compared with the CCI group, the PWMT and PWTL of the CCI + MMP-9 group at 1, 2, and 3 days postoperatively both decreased $(\mathrm{F}=11.90, \mathrm{P}=0.000 ; \mathrm{F}=11.57, \mathrm{P}=0.000)$, and there was significant differences at 1 day after CCI $(\mathrm{t}=6.914$, $\mathrm{P}=0.000 ; \mathrm{t}=3.993, \mathrm{P}=0.000)$. In the CCI + TIMP-1 group, the PWMT and PWTL increased at 1, 2, and 3 days after CCI surgery $(\mathrm{F}=10.71, \mathrm{P}=0.001 ; \mathrm{F}=11.67, \mathrm{P}=0.000)$. The PWMT and PWTL at 1 day after surgery were respectively
$77 \%$ and $69 \%$ of the basal pain threshold, and compared with the basal pain threshold, the difference was significant $(\mathrm{t}=3.648, \mathrm{P}=0.000 ; \mathrm{t}=3.170, \mathrm{P}=0.034)$. Meanwhile, in the CCI + siRNA group, the PWMT and PWTL increased at $1,2,3$, and 5 days after $\mathrm{CCI}$ surgery $(\mathrm{F}=10.46, \mathrm{P}=0.000$; $\mathrm{F}=10.26, \mathrm{P}=0.000)$. The PWMT and PWTL at 1 day after the CCI operation were $87 \%$ and $98 \%$ of the basal pain threshold, and compared with the basal pain threshold, the difference was significant $(t=5.008, P=0.000 ; t=7.882$, $\mathrm{P}=0.034)$. The difference in the decline rate between the two groups can indicate that the CCI + siRNA group is more efficient than the CCI + TIMP-1 group. The above data shows that MMP-9 can significantly aggravate mechanical and thermal hyperalgesia in CCI rats at 1 day after surgery (Figure 1).

\section{The expressions of MMP-9 and CX3CL1 protein in the DRG of the spinal cord}

The WB results show that compared with the Sham group, the expressions of MMP-9 and CX3CL1 protein of the CCI group at 1 day after CCI surgery were significantly increased $(\mathrm{F}=21.457, \mathrm{P}=0.000 ; \mathrm{F}=20.001, \mathrm{P}=0.000)$ (Figure 2); in contrast, the expressions of MMP-9 and CX3CL1 protein of the CCI + MMP-9 group increased $(\mathrm{F}=80.435$, $\mathrm{P}=0.000 ; \mathrm{F}=56.554, \mathrm{P}=0.000)$ at 1 day after operation, while the expressions of the MMP-9 and CX3CL1 protein was significantly reduced in the CCI + TIMP-1 group $(\mathrm{F}=11.056, \mathrm{P}=0.024 ; \mathrm{F}=6.056, \mathrm{P}=0.035)$ (Figure 3); the expressions of MMP-9 and CX3CL1 protein of the CCI + siRNA group was reduced $(\mathrm{F}=71.225, \mathrm{P}=0.000 ; \mathrm{F}=56.241$, $\mathrm{P}=0.000$ ) (Figure 4); compared with the CCI + TIMP-1 group, the expressions of MMP-9 and CX3CL1 protein of the CCI + siRNA group were reduced at 1 day after CCI $(\mathrm{F}=17.35, \mathrm{P}=0.000 ; \mathrm{F}=13.180, \mathrm{P}=0.017$ ) (Figure 5). These data indicate that the expression of CX3CL1 protein in the CCI rats increases and that the change of MMP-9 is closely related to CX3CL1. Combined with the results of the pain threshold changes (Figure 1), the CCI + MMP-9 group had significantly decreased at 1 day after operation, compared with the CCI group, and there was a significant difference between PWMT and PWTL $(\mathrm{t}=6.914, \mathrm{P}=0.000 ; \mathrm{t}=3.993$, $\mathrm{P}=0.000)$. There was a significant difference between PWMT and PWTL in the CCI + TIMP-1 group at 1 day postoperatively $(\mathrm{t}=3.648, \mathrm{P}=0.000 ; \mathrm{t}=3.170, \mathrm{P}=0.034)$. The PWMT and PWTL at 1 day postoperatively was $77 \%$ and $69 \%$ of the basal pain thresholds, respectively, while the PWMT and PWTL of the CCI + siRNA group 

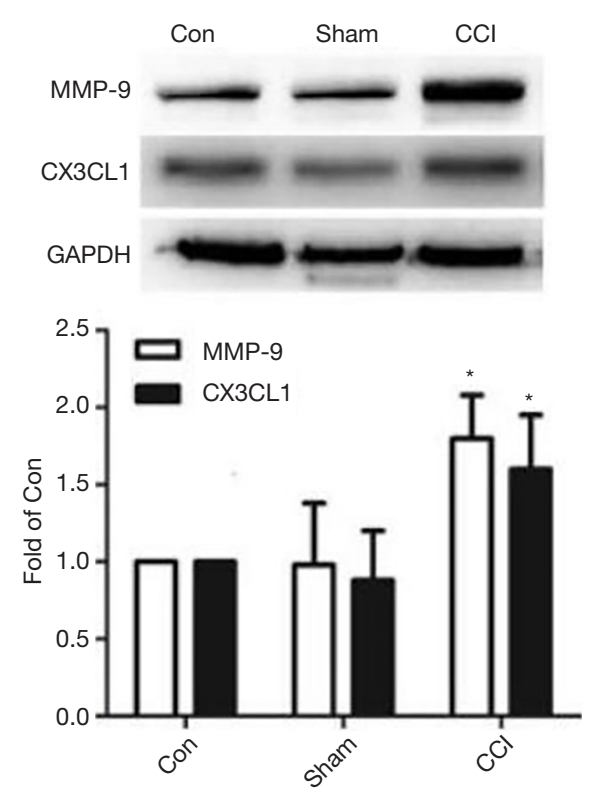

Figure 2 The expressions of MMP-9 and CX3CL1 protein in spinal DRG 1 day after surgery. The data are presented as mean \pm $\mathrm{SD}$ in each group $(\mathrm{n}=6)$. * $\mathrm{P}<0.01$ vs. Sham group.
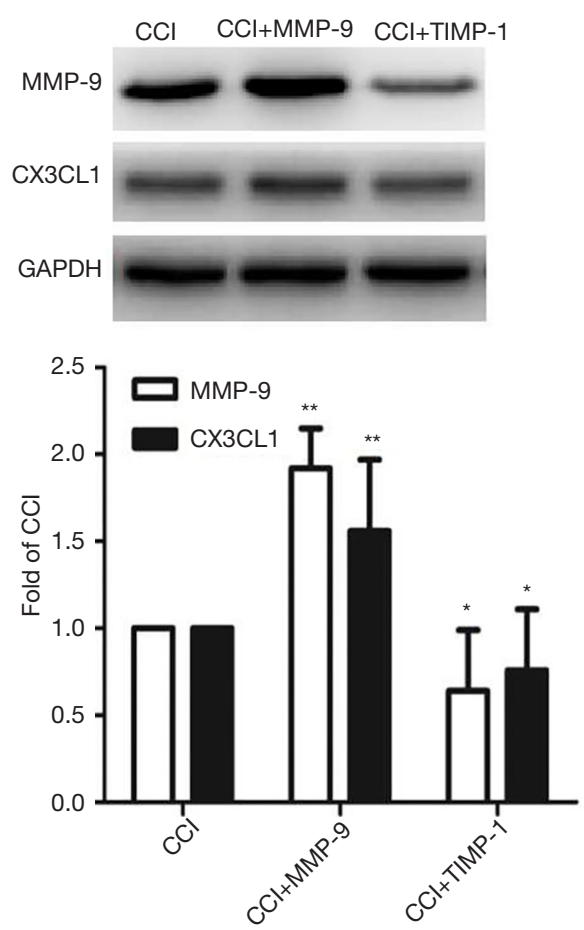

Figure 3 The expressions of MMP-9 and CX3CL1 protein in spinal DRG $1 \mathrm{~d}$ after surgery. The data are means $\pm \mathrm{SD}$ in each group ( $\mathrm{n}=6) .{ }^{*}, \mathrm{P}<0.05,{ }^{* *}, \mathrm{P}<0.01$ vs. CCI group. CCI, chronic sciatic nerve constriction injury.
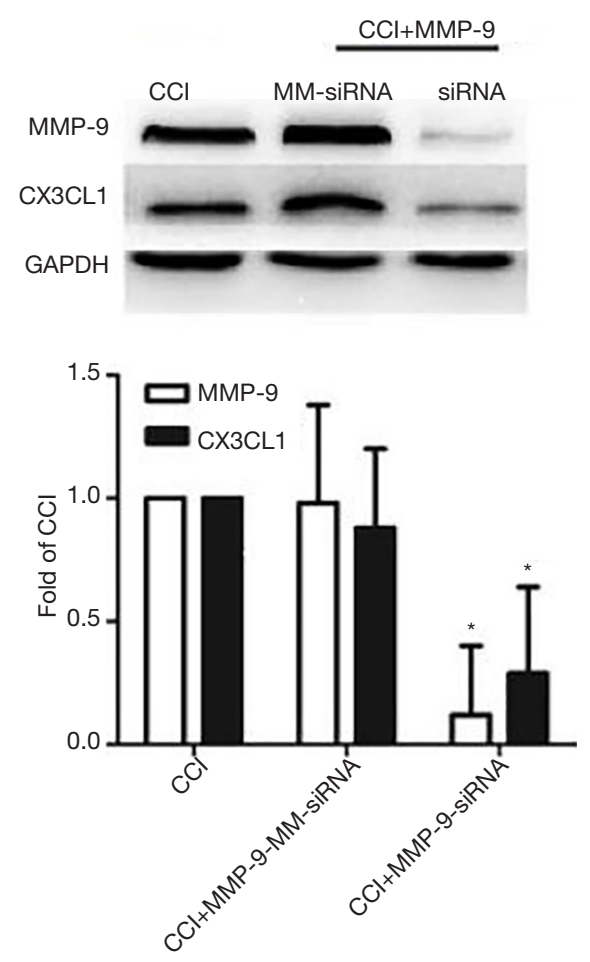

Figure 4 The expressions of MMP-9 and CX3CL1 protein in spinal DRG 1 day after surgery. The data are presented as mean $\pm \mathrm{SD}$ in each group $(\mathrm{n}=6)$. ${ }^{*}, \mathrm{P}<0.01$ vs. CCI group. CCI, chronic sciatic nerve constriction injury.

at 1 day postoperatively were $87 \%$ and $98 \%$ of the basal pain threshold, respectively. These findings indicate that, regardless of whether pharmacological methods antagonize the effect of MMP-9 (inhibitor TIMP-1) or molecules biologically (MMP-9-siRNA gene silencing) downregulate the expression of MMP-9, the content and activity of MMP-9, the CX3CL1 of neuron surface abscission and activation, and the pain sensitive response of CCI rats can be effectively reduced. However, gene-silencing technology is more efficient than intrathecal injection of inhibitors, and it is more targeted to MMP-9.

\section{Discussion}

NP involves both the central and peripheral nervous system. With the introduction of pain sensations, a series of changes occur in the neurons and glial cells of the central nervous system, releasing neurotransmitters and modulators, producing and consolidating this pain signal. 


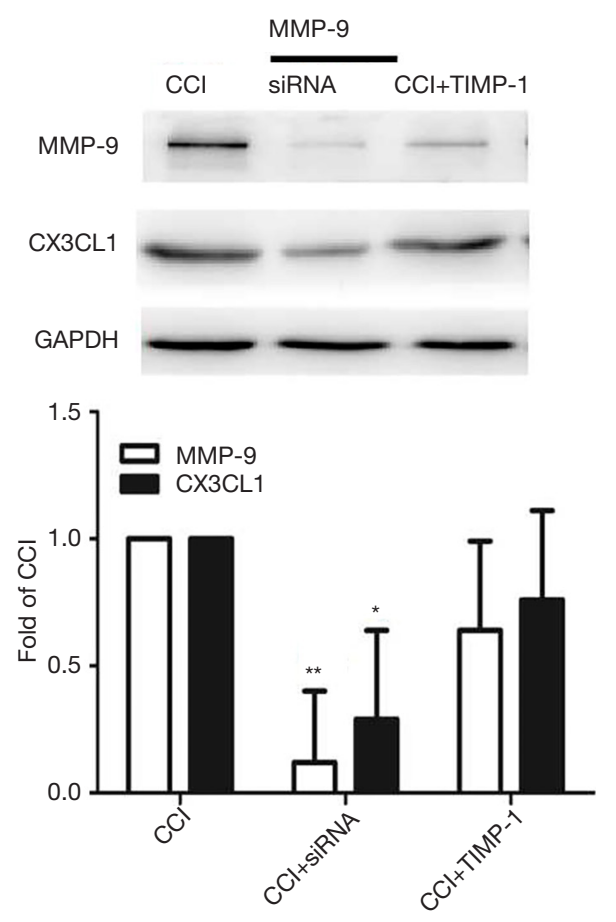

Figure 5 The expressions of MMP-9 and CX3CL1 protein in spinal DRG 1 day after surgery. The data are presented as mean \pm $\mathrm{SD}$ in each group $(\mathrm{n}=6) .{ }^{*}, \mathrm{P}<0.05,{ }^{* *}, \mathrm{P}<0.01$ vs. CCI + TIMP-1 group. CCI, chronic sciatic nerve constriction injury.

Matrix metalloproteinases (MMPs) are a group of zinc ion-dependent peptide endonucleases, which have many subtypes. MMP-9 and MMP-2 are mainly expressed in primary sensory neurons (10), which are more commonly studied in chronic pain. Studies show that MMP-9 and MMP-2 play roles in the early and late stages of chronic nerve injury, respectively. MMP-9 is a proteolytic enzyme, also known as gelatinase $\mathrm{B}$, which can be produced by various cells such as macrophages and neutrophils. It can act on chemokines (such as SDF-1 and SCF), adhesion molecules (such as cadherin and ICAM-5) and participate in pathophysiological processes such as inflammatory response and angiogenesis (4). It was found that the downregulation and inhibition of mmp-9 could reduce the dieback of axons in cultured neurons in vivo and in vitro, and this has been used in the treatment of spinal cord injury. In addition, fulminomycin (the specific inhibitor of MMP-9), minocycline, and methylprednisolone have similar effects $(11,12)$. This experiment found that the intrathecal injection of MMP-9 decreased the pain threshold in CCI model rats, especially at 1 day postoperatively. Meanwhile, for intrathecal injection of TIMP-1 at 1 day postoperatively, the pain threshold of rats increased significantly in the CCI rat, indicating that MMP-9 plays a role in the early phase of $\mathrm{NP}$ and exacerbates the pain response in CCI rats, which is in line with another study in which expression of MMP-9 in DRG was consistent with changes in the early phase of NP (3).

RNA interference technology is a common method used to control gene expression study and gene function, to develop new therapeutic drugs, and to improve the treatment of chronic pain (13). Meanwhile, siRNA technology can design precise targeted therapy for protein genes with known structural information, which can more directly and efficiently study the role of specific proteins. Intrathecal injection of siRNA and certain transfection reagents (such as lipid transfection amines and polyethyleneimine) have been shown to knock out gene expression in the DRG and spinal cord to modulate pain sensitivity (14). The results of this experiment show that pre-intrathecal administration of MMP-9-siRNA can reverse the effect of MMP-9, but administration of MM-siRNA has no similar effect on MMP-9 (Figure 4), indicating that intrathecal injection of MMP-9-siRNA effectively inhibited the expression of MMP-9 in the DRG, further demonstrating that this experimental model was successful. The PWMT and PWTL in the CCI + TIMP1 group at 1 day postoperatively were $78 \%$ and $70 \%$ of the basal pain threshold, respectively; the PWMT and PWTL in the CCI + siRNA group were $87 \%$ and $98 \%$ of the basal pain threshold, respectively; compared with the rats in the CCI + TIMP-1 group, the expressions of MMP-9 and CX3CL1 protein in the CCI + siRNA group was reduced 1 day after CCI surgery $(\mathrm{F}=17.35, \mathrm{P}=0.000 ; \mathrm{F}=13.180$, $\mathrm{P}=0.017$ ) (Figure 5), which illustrates that the siRNA technology can inhibit the expression of $M M P-9$ gene with higher efficiency, further indicating that MMP-9 acts in the early stage of CCI rats by regulating the expression of CX3CL1 protein.

CX3CL1 is the only member of the known chemokine CX3CL subfamily. It mainly exists on the surface of neurons in brain and DRG of the spinal cord. The expression level of CX3CLl changes due to central nervous system damage and neuroinflammation (15). CX3CLl exists in two forms: membrane-bound (inactive state) and soluble (active state). The balance between them is regulated by metalloproteinase-dependent proteases $(16,17)$. Some scholars believe that CX3CL1 can be released directly from the cell membrane under stress (no protein synthesis 
time is required). This may be a new rapid way to adjust the concentration of substances in local areas, but it is not known whether there is protease that acts on the CX3CLl on the surface of neurons to make it soluble to CX3CL1, so as to bind to its specific receptor and then play a role in NP. The results of this study show that compared with the CCI group, the expressions of MMP-9 and CX3CL1 protein in the CCI + MMP-9 group rats were significantly increased at 1 day after CCI operation, while the expressions of MMP-9 and CX3CL1 protein in the CCI + TIMP-1 group at the same time point were reduced. The synchronous changes of MMP-9 and CX3CL1 in the DRG of spinal cord in CCI rats are closely related to changes in the pain threshold of rats, suggesting that MMP-9 plays a role in early phase of $\mathrm{NP}$, and its role may be mediated by CX3CL1.

Our experimental results show that the activity of MMP-9 and CX3CL1 significantly increased at 1 day after CCI surgery (the early phase of NP). It is speculated that MMP-9, perhaps similarly to MMP-2 (10), sheds from the surface of neurons and becomes soluble to CX3CL1, and then acts on the receptor CX3CR1 to participate in the next cascade reaction and participate in the early formation of NP. Studies have shown that microglia participate in the early initiation phase of NP, and astrocytes participate in the later maintenance phase of NP $(18,19)$, but whether MMP-9 is secreted by activated microglia in the CCI rat model is still unclear and the subsequent effect on CX3CL1 needs to be confirmed by further research.

MMP-9 is involved in the early phase of NP in CCI rats. The mechanism functions by regulating the expression of CX3CL1/CX3CR1. Intervention of MMP-9 is expected to become a therapeutic target in the early stage of NP.

\section{Acknowledgments}

Funding: This research was supported by grant from the Natural Science Foundation of Zhejiang Province (LY17C090002) and General Medical and Health Project of Zhejiang Province ( 2016KYA156).

\section{Footnote}

Reporting Checklist: The authors have completed the ARRIVE reporting checklist. Available at http://dx.doi. org/10.21037/apm-20-1078

Data Sharing Statement: Available at http://dx.doi. org/10.21037/apm-20-1078
Conflicts of Interest: All authors have completed the ICMJE uniform disclosure form (available at http://dx.doi. org/10.21037/apm-20-1078). LZ reports grants from General Medical and Health Project of Zhejiang Province, during the conduct of the study; JS reports grants from Natural Science Foundation of Zhejiang Province, during the conduct of the study. The other authors have no conflicts of interest to declare.

Ethical Statement: The authors are accountable for all aspects of the work in ensuring that questions related to the accuracy or integrity of any part of the work are appropriately investigated and resolved. The procedures of animal experiments were performed by Guide for the Care and Use of Laboratory Animals. The study was approved by the Zhejiang Chinese Medical University, China (animal quality certificate number: SCXK, Shanghai, 2012-0012; experimental breeding room license number: SYXK, Zhejiang, 2003-0003).

Open Access Statement: This is an Open Access article distributed in accordance with the Creative Commons Attribution-NonCommercial-NoDerivs 4.0 International License (CC BY-NC-ND 4.0), which permits the noncommercial replication and distribution of the article with the strict proviso that no changes or edits are made and the original work is properly cited (including links to both the formal publication through the relevant DOI and the license). See: https://creativecommons.org/licenses/by-nc-nd/4.0/.

\section{References}

1. Sewell RD. Neuropathic pain models and outcome measures: a dual translational challenge. Ann Transl Med 2018;6:S42.

2. Yao J, Zhang L, Lei W, et al. Intra-amygdala infusion of zeta inhibitory peptide attenuates neuropathic pain but not inflammatory pain in adult rats. Ann Palliat Med 2019;8:660-6.

3. Kawasaki Y, Xu ZZ, Wang X, et al. Distinct roles of matrix metalloproteases in the early-and late-phase development of neuropathic pain. Nat Med 2008;14:331-6.

4. Lu B, Yao J, Lei WP, et al. Role of the CX3CR1/ ERK5 pathway in spinal microglia for the development of neuropathic pain. Chinese Medical Journal 2013;93:1997-2000.

5. Sun JL, Xiao C, Lu B, et al. CX3CL1/CX3CR1 Regulates nerve injury-induced pain hypersensitivity through the 
ERK5 signaling pathway. J Neurosci Res 2013,91:545-53.

6. Yang JP, Jiang H. Improvement of long-term indwelling operation of subretinal tube embedding in rats. Chinese Journal of Anesthesiology 1993;13:110-2.

7. Du S, Bao X, Feng M, et al. A Device of micro-catheter protection for chronic lumbar catheterization of the spinal subarachnoid space in rats. Chinese Journal of Comparative Medicine 2011;(3):52-5.

8. Bennett GJ, Xie YK. A peripheral mono-neuropathy in rat that produces disorders of pain sensation like those seen in man. Pain 1988;33:87-107.

9. He DD, Guo XY. The cAMP-PKA signaling pathway mediates the production and maintenance of bone cancer pain in rats. Chinese Journal of Pain Medicine 2015;21:10-4.

10. Dobrie LA. The Effects of Matrix Metalloproteinase-9 on CX3CL1 Shedding and Axon Retraction. 2019. Available online: https://stars.library.ucf.edu/honorstheses/506

11. Busch SA, Horn KP, Silver DJ, et al. Overcoming macrophage-mediated axonal dieback following CNS injury. J Neurosci 2009;29:9967-76.

12. Evans TA, Barkauskas DS, Myers JT, et al. Highresolution intravital imaging reveals that blood-derived macrophages but not resident microglia facilitate secondary axonal dieback in traumatic spinal cord injury. Exp Neurol 2014;254:109-20.

Cite this article as: Zhao L, Song C, Huang Y, Lei W, Sun J. MMP-9 regulates CX3CL1/CX3CR1 in the early phase of neuropathic pain in chronic sciatic nerve constriction injury (CCI) rats. Ann Palliat Med 2020;9(4):2020-2027. doi: 10.21037/apm-20-1078
13. Soutschek J, Akinc A, Bramlage B, et al. Therapeutic silencing of an endogenous gene by systemic administration of modified siRNAs. Nature 2004;432:173-8.

14. Tan PH, Gao YJ, Berta T, et al. Short small-interfering RNAs produce interferon- $\alpha$-mediated analgesia. Br J Anaesth 2012;108:662-9.

15. Poniatowski ŁA, Wojdasiewicz P, Krawczyk M, et al. Analysis of the role of CX3CL1 (Fractalkine) and its receptor CX3CR1 in traumatic brain and spinal cord injury: Insight into recent advances in actions of neurochemokine agents. Mol Neurobiol 2017;54:2167-88.

16. Ragozzino D. CXC chemokine receptors in the central nervous system: Role in cerebellar neuromodulation and development. J Neurovirol 2002;8:559-72.

17. Kerfoot SM, Lord SE, Bell RB, et al. Human fractalkine mediates leukocyte adhesion but not capture under physiological shear conditions; a mechanism for selective monocyte recruitment. Eur J Immunol 2003;33:729-39.

18. Zhuang ZY, Gerner P, Woolf CJ, et al. ERK is sequentially activated in neurons, microglia, and astrocytes by spinal nerve ligation and contributes to mechanical allodynia in this neuropathic pain model. Pain 2005;114:149-59.

19. Mika J, Zychowska M, Popiolek-Barczyk K, et al. Importance of glial activation in neuropathic pain. Eur J Pharmacol 2013;716:106-19. 